\title{
Surgical Technique of Endoscopic Transforaminal Decompression and Fusion with a Threaded Expandable Interbody Fusion Cage and A Report of 24 Cases
}

\section{Kai-Uwe Lewandrowski*}

Department of Orthopaedic Spine Surgery, Center for Advanced Spine Care of Southern Arizona, USA

"Corresponding author: Kai-Uwe Lewandrowski, Department of Orthopaedic Spine Surgery, Center for Advanced Spine Care of Southern Arizona, USA, Tel: 5202041495, E-mail: business@tucsonspine.com

Rec Date: March 03, 2018; Acc Date: March 06, 2018; Pub Date: March 09, 2018

Copyright: ( 2018 Lewandrowski KU. This is an open-access article distributed under the terms of the creative commons attribution license, which permits unrestricted use, distribution, and reproduction in any medium, provided the original author and source are credited.

\section{Abstract}

Fusion of the anterior column with placement of interbody fusion cages is commonly employed in the treatment of instability-related degenerative disc disease that has been proven refractory in non-operative treatment. Interbody fusion cages aid in the containment of bone graft, promotion of more reliable fusion, and restoration of neuroforaminal height. The VariLift ${ }^{\circledR}$ Interbody Fusion System (VariLift ${ }^{\circledR}$ system) has been developed as a standalone solution to address subsidence and migration problems seen with traditional lumbar interbody fusion cages that require posterior supplemental transpedicular screw fixation for improved stability. This series of 24 consecutive patients describes patient selection criteria and the technical aspects of this novel endoscopic transforaminal lumbar interbody fusion technique in a step-by-step fashion. The transforaminal endoscopically assisted interbody fusion with the standalone expandable VariLift ${ }^{\circledR}$ system represents a simplified alternative to other pedicle screw-based spinal fusion.
\end{abstract}

Keywords: Biomechanics engineering; Endoscopic interventional surgery; Interbody fusion

\section{Introduction}

Minimally invasive percutaneous lumbar decompression and fusion procedures using small tubular or bladed retractor systems have become commonplace in the treatment of instability-related, symptomatic disc degeneration in patients who have failed nonoperative care. These procedures have resulted in drastically reduced hospitalization stays with a lower rate of medical complications [1,2]. Additional advantages include lower blood loss, less postoperative pain, lower anxiety levels, and improved mobility [3]. The implication is that use of surgical procedures that do not require extensive muscle stripping, or aggressive resection of the lumbar posterior elements, are associated with a lower incidence of peri- and possibly long-term postoperative problems [4,5].

Minimally invasive reconstructive anterior column procedures with placement of interbody fusion cages allow stabilization of the diseased lumbar spinal motion segment, while preserving neuroforaminal height and promoting bony ingrowth [6]. Interbody fusion with conventional non-expandable cages typically requires endplate decortication, impaction of the implant into the interspace, and posterior supplemental fixation with pedicle screws to avoid migration or subsidence of the cage and loss of lordosis [7-9]. The additional use of pedicle screw instrumentation clearly increases operative time, blood loss, and has been associated with higher complication rates due to intraoperative nerve root injury, damage to the adjacent facet joint complex $[10,11]$, and propagation of symptomatic adjacent segment disease [12].

The percutaneous endoscopic transforaminal decompression and fusion procedure described in this surgical technique guide takes advantage of proven benefits of minimal muscle dissection and minimal spinal instrumentation. It employs an expandable interbody fusion system (VariLift $-\mathrm{L}$ ) designed as a stand-alone expandable fusion device. The device maintains lumbar lordosis and resists subsidence and migration. Its large chamber provides ample room for bone graft and the wide fenestrations allow radiographic assessment of postoperative spinal fusion progression. This low-profile device is ideally suited for endoscopically assisted transforaminal implantation as it neither requires impaction, nor aggressive decortication of the endplates. After foraminoplasty, the threaded device can be placed percutaneously over a guide wire into the intervertebral disc space. It is easily advanced by turning it clockwise and retrieved by turning it counterclockwise with solid purchase to the endplates over a large contact area without violating the endplates. In situ expansion induced ligamentotaxis of the remaining annulus fibrosus renders the motion segment instantly biomechanically stable at the time of implantation without the need for any supplemental fixation.

The VariLift -L implant has been employed during PLIF and TLIF with either two smaller cages during PLIF [13-15], or one larger obliquely placed cage during TLIF [15]. The percutaneous endoscopic transforaminal implantation is made feasible by more advanced spinal endoscopes and decompression tools such as reamers, trephines, motorized shavers, osteotomes, and rongeurs. These instruments afford better videoendoscopic visualization and tissue dissection, as well as more effective decompression maneuvers that were hitherto not possible. Surgical indications with spinal endoscopy have broadened, therefore, and are no longer limited to herniated disc. This surgical technique guide provides a description of the steps required to perform a stand-alone percutaneous endoscopically assisted transforaminal interbody fusion surgery. 


\section{Patients and Selection Criteria}

In 2007, the Center for Advanced Spine Care of Southern Arizona established an outpatient spinal surgery program for the treatment of lumbar herniated disc and spinal stenosis. With the advancement of videoendoscopic equipment, bony decompression became more feasible broadening the indication to treat spinal stenosis. These advancements provided the foundation for the development of a fullendoscopic decompression procedure popularized by Ruetten et al., which essentially is a combination of the posterolateral transforaminal and the direct posterior interlaminar access to the lumbar neuroforamen and lateral recess allowing combined decompression of neural elements posteriorly and anteriorly [16-19]. The combination of these advanced percutaneous decompression techniques with an expandable threaded interbody fusion device allowed further expansion of the indication of the outpatient percutaneous transforaminal procedure to treat instability-induced symptoms of degenerated disc disease that have proven refractory to non-operative treatment - the main indication for this procedure. Patient inclusion criteria are:

1. Clinical signs of lumbar radiculopathy, dysesthesias, and decreased motor function;

2. Imaging evidence of foraminal or lateral recess stenosis demonstrated on preoperative magnetic resonance images (MRI) and computed tomography (CT) scans;

3. Grade I spondylolisthesis;

4. Unsuccessful non-operative treatment, including physical therapy and transforaminal epidural steroid injections for at least 12 weeks; and

5. An age of $35-85$ years.

Patients considered not suitable for the transforaminal endoscopically assisted intervertebral fusion procedure were stratified according to the following exclusion criteria:

1. Segmental instability with greater than Grade I spondylolisthesis or translational motion of greater than $8 \mathrm{~mm}$ on preoperative extension flexion radiographs;

2. Severe central stenosis (less than $100 \mathrm{~mm}^{2}$ ) [20];

3. Extensive facet arthropathy;

4. Infection; and

5. Metastatic disease.

All patients in this consecutive case series of 24 patients (14 females and 10 males) provided informed consent.

\section{Step-by-Step Surgical Techniques}

The surgical procedure employs the endoscopic transforaminal approach using the "outside-in" technique, in which the working sheath is placed into the lower portion of the neuroforamen, thus, retracting and avoiding the exiting nerve root. No part of the cannula tip or the endoscope is positioned in the disc space. The surgical technique, originally popularized by Hoogland and Schubert et al., $[21,22]$ employs an expansile foraminoplasty in patients with foraminal and lateral stenosis.

Procedures are performed in prone position under general anesthesia with adjunctive use of local anesthesia using $0.25 \%$ bupivacaine in all patients. In some instances, where access to the L5/S1 neuroforamen is difficult due to a high riding ilium, patients can be positioned in the lateral decubitus position. Techniques to define the skin entry point and the surgical trajectory have been described elsewhere [23-30]. Entry points are generally laterally at 7-9 $\mathrm{cm}$ at the L3/L4 level, $8-10 \mathrm{~cm}$ at the L4/L5 level, and $10-12 \mathrm{~cm}$ at the L5/S1 level. The targeted neuroforamen is accessed as follows:

\section{Step 1: Needle placement}

An 18-G (150 mm in length) needle is inserted into the safe zone of Kambin's triangle bordered by the traversing nerve root medially, the exiting nerve root laterally, and the lower adjacent pedicle distally $[27,28]$. Ideally, the targeting needle was placed on the lateral view into the lower portion of the neuroforamen or into the disc. On the anterior-posterior view, the needle tip should be at the medial interpedicular line. A steel guide wire was then inserted and the $18-\mathrm{G}$ spinal needle was removed.

\section{Step 2: Placement of working cannula}

Dilators, drills, and trephines of increasing diameters were used for foraminal decompression procedures. Additional cannulated reamers measuring $7 \mathrm{~mm}$ and $9 \mathrm{~mm}$ in diameter intended to be used over a guide wire without the protective working cannula were available but rarely used to further minimize risk of dysesthesia of the exiting nerve root and irritation of its dorsal root ganglion (Figure 1).

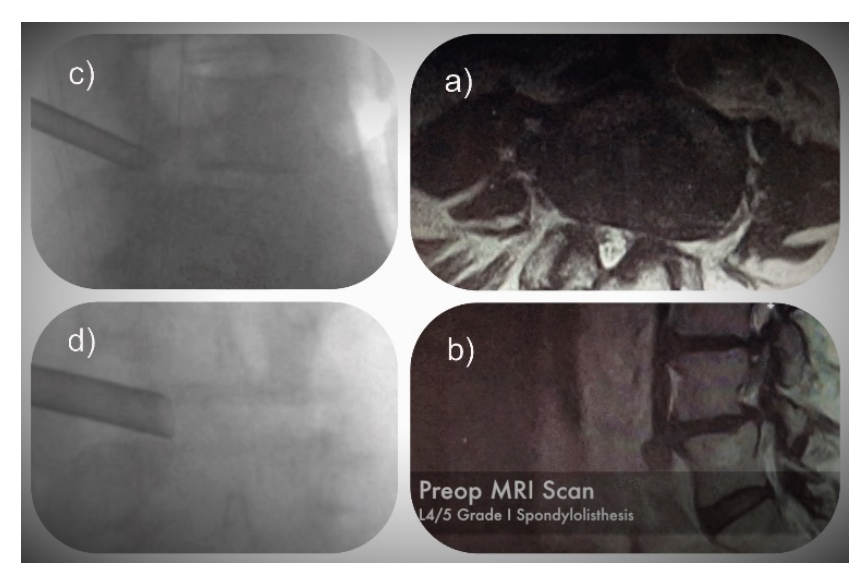

Figure 1: Pre-operative axial (a), and sagittal (b) MRI scan of an 80year-old female patient with spondylolisthesis at L4/L5 and lateral recess and foraminal stenosis (Case I). A transforaminal working cannula was placed at the lateral recess as shown in the lateral (c), and in the anterior-posterior (a) projection.

At this point, the integrity of the intervertebral disc tissue is tested by attempting to advance a pituitary rongeur into the presumably hollow disc space (Figure 2). Once confirmed fluoroscopically, the vertical height restoration interbody fusion procedure can commence as described in the following.

\section{Step 3: Foraminoplasty and SAP resection}

Endoscopic osteotomes, motorized drills, Kerrison rongeurs, and percutaneous trephines were employed through the inner $4.1 \mathrm{~mm}$ inner working channel of the spinal endoscope for the foraminoplasty, which was done via removal of bone from the hypertrophied superior and inferior articular process (Figure 3). 

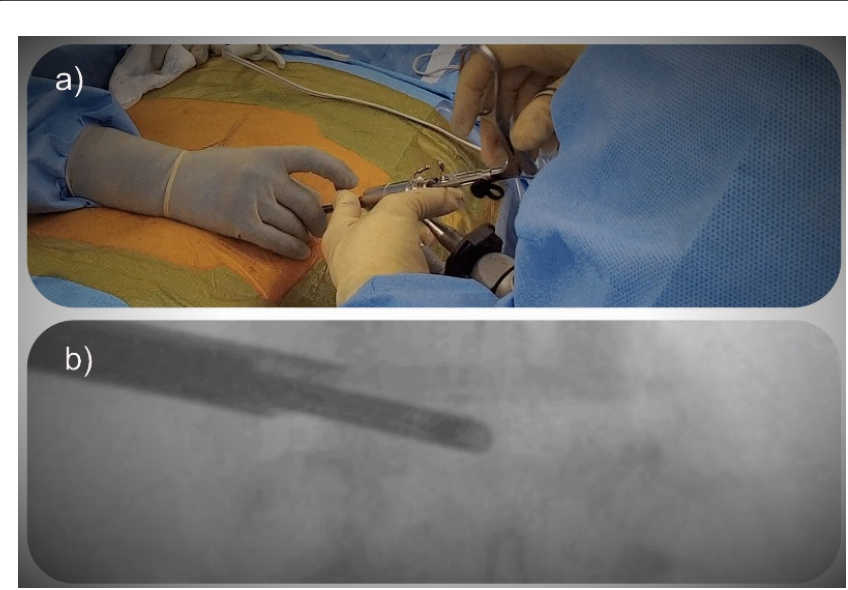

Figure 2: (a) Transforaminal decompression with a spinal endoscope. (b) Intraoperative probing of the intervertebral disc showing a void disc space.
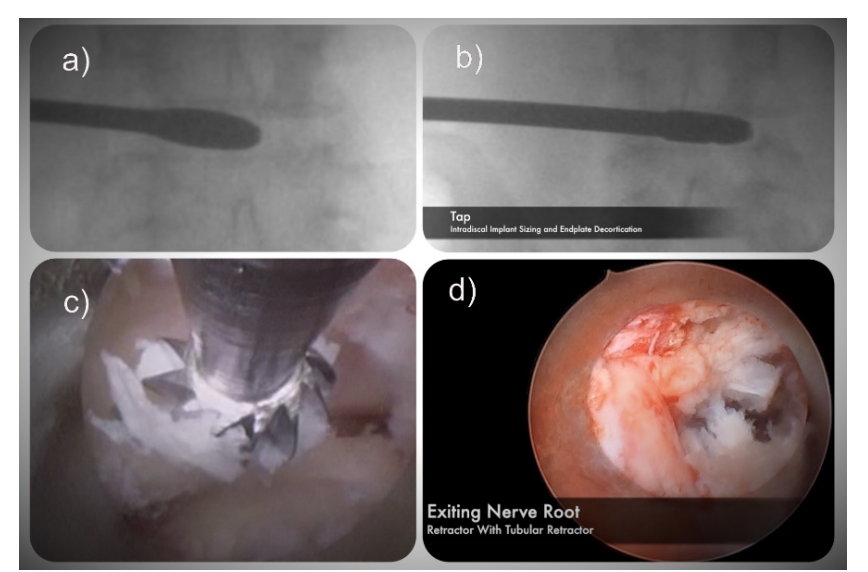

Figure 3: (a) Anterior posterior fluoroscopy view of a paddle shaver placed percutaneously through the transforaminal approach into the intervertebral disc space to decorticate the endplates. (b) Placement of threaded tap to prepare insertion of the threaded fusion cage. (c) Transforaminal decompression of the superior articular process of the lumbar facet joint to gain access to the lateral recess using a drill. (d) Completed decompression of the exiting nerve root and preparation of the intervertebral space ready for insertion of the threaded VariLift ${ }^{\oplus}$ L implant.

The entire superior articular process (SAP) was resected starting rostral to distal via osteotomy and detached from the superomedial pedicle wall. Endoscopic drills, osteotomes, and rongeurs were deployed inside the inner working channel of the endoscope to lessen the risk of dysesthesia and irritation of the exiting nerve root and its dorsal root ganglion. In other words, the entire decompression was performed under continuous direct videoendoscopic visualization and no part of the decompression procedure was done percutaneously with fluoroscopic imaging only. Local bone graft harvested during this part of the procedure should be collected and saved for the interbody fusion (see below).

\section{Step 4: Partial pediculectomy}

The foraminoplasty was expanded by changing the trajectory of the instruments to aim for inferior pedicle. The superiomedial pedicle wall of the distal pedicle was partially resected. This is often necessary to prepare the introitus for the expandable cage to promote parallel alignment of the implant with the vertebral interspace and to avoid rostral migration of the cage insertion point into the axilla between the exiting and traversing nerve root. The cortical bone of the superomedial pedicle wall is typically denser than the bone of the superior ring apophysis and should, therefore, be partially resected for parallel access and to prevent the implant from cutting preferentially into the superior endplate. Decompression below the traversing nerve root can be completed by drilling down the inferior ring-apophysis and any central disc bulge below the traversing nerve root, if necessary. In case of concomitant herniated disc, extruded disc material can easily be removed using forceps and pituitary rongeurs and contained herniations can be decompressed through a small annular window. Epidural bleeding can be controlled with a radiofrequency probe (Ellman ${ }^{\circledR}$; Ellman International LLC, USA) under saline irrigation.

\section{Step 5: Discectomy and interspace preparation}

An annulotomy is performed to access the intervertebral disc space. Paddle shavers are used to remove disc tissue and to decorticate the endplates (Figure 3). Care should be taken to not violate the endplates through the subchondral bone to minimize implant subsidence. Therefore, paddle shavers and other instruments used for the discectomy should be lined up parallel to the endplate. Pituitary rongeurs can be used to remove disc tissue through the central working channel of the spinal endoscope. The entire disc space can be debrided by advancing or withdrawing the endoscope and by moving it from posteriorly to anterior by raising one's hand. Rotating the endoscope's $20^{\circ}$ lateral view optic also allows direct visualization of the endplates during the decortication and discectomy maneuvers.

\section{Step 6: Sizing and taping}

The VariLift - - interbody fusion system comes with many interbody shavers and taping sizers. They start at $10 \mathrm{~mm}$ to $14 \mathrm{~mm}$ with the final expanded outer diameter typically being one $\mathrm{mm}$ larger than its starting size prior to the expansion maneuver. The sizing taps have a conical design to ease insertion into the interspace. The outer diameter is $4 \mathrm{~mm}$ smaller at the tip than at the end of the implant's body. Under fluoroscopic guidance, the sizing tap is inserted into the intervertebral disc space by turning it clockwise. The ideal starting point is within Kambin's safe zone between the medial and lateral pedicle wall in the axial plane. The posterolateral insertion trajectory in the axial plane is typically between 40 to 60 degrees of the mid-sagittal vertical plumb line but essentially dictated by the patient's vertical alignment of the intervertebral discs in relation to the iliac crest. Attention should be paid to excessively high access angles in patients with advanced vertical spinal collapse and sacralization of the L5 vertebral body where the access can be obstructed inferiorly by a large sacral alar and laterally by a hypertrophic arthritic facet joint (see L5/S1 fusion pearls). The sizing tap should be snug and a good fit can easily be assessed by attempting to either remove the tap or moving it side to side. After final sizing is accomplished, the tap can easily be removed by turning it counterclockwise (Figure 3). 
Citation: Lewandrowski KU (2018) Surgical Technique of Endoscopic Transforaminal Decompression and Fusion with a Threaded Expandable Interbody Fusion Cage and A Report of 24 Cases. J Spine 7: 409. doi:10.4172/2165-7939.1000409

Page 4 of 8

\section{Step 7: Bone grafting}

Local bone graft should be inserted via the working sleeve of the spinal endoscope or through its inner working channel under direct visualization. Abundant bone graft should be deployed and impacted into the periphery of the interspace in a circumferential manner leaving the central area without bone graft to accommodate the VariLift ${ }^{\oplus}$ - $L$ cage. This can be assured by inserting the last paddle shaver once more and rotating it several times. The bone grafting can also be done under videoendoscopic control.

\section{Step 8: Cage insertion and expansion}

The cage can be inserted with its cannulated inserter device via a nitinol guide wire that was previously placed into the interspace through the central working channel of the spinal endoscope.

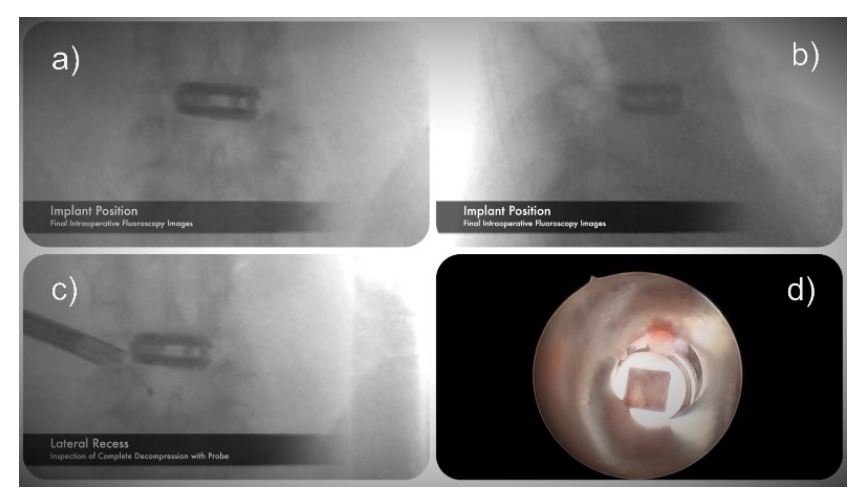

Figure 4: Intraoperative endoscopically assisted direct visualization of the endplate preparation (a), and the intervertebral disc space (b). The previously cut threads are cut into the decorticated endplates. The VariLift ${ }^{\circ} \mathrm{L}$ implant is inserted via a nitinol guide wire obliquely into the midportion of the intervertebral disc space as shown here in the anteroposterior ( $c$ and e), and the lateral ( $d$ and f) fluoroscopic projection.

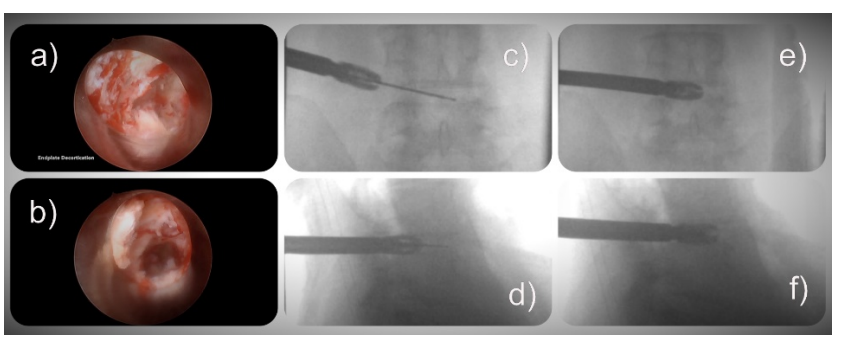

Figure 5: Intraoperative view of the percutaneous insertion of the VariLift'-L implant mounted on the inserter. The VariLift ${ }^{\circ}-\mathrm{L}$ implant is expanded by turning the center expansion set screw clockwise via the inserter's central working channel (a). Full expansion is achieved once the center expansion set screw is tight. (b) Bone graft, in this case demineralized allograft bone matrix, can be inserted through the same inner working channel of the inserter with use of a funnel and push rod system. The bone graft is extruded through the lateral fenestrations of the VariLift -L implant into the intervertebral disc space.
Once the VariLift - $\mathrm{L}$ interbody fusion cage is engaged in between the two adjacent endplates, it can be inserted into the interspace by turning the inserter clockwise until the implant reaches its desired midline position in the anterior-posterior as well as in the lateral fluoroscopic view (Figure 4). The implant should be sufficiently recessed by approximately $3 \mathrm{~mm}$ below the annulus. The central expansion screw is then driven forward by turning it clockwise with its driver being inserted through the cannulated cage inserter (Figure 5).

Once maximum expansion is reached, additional bone graft can be inserted through the central chamber of the VariLift $-\mathrm{L}$ cage and extruded into the interspace via the lateral fenestrations of the implant (Figure 5b). The end cap is then placed and tightened before the VariLift $-\mathrm{L}$ cage is detached and withdrawn through the percutaneous posterolateral incision (Figure 6).

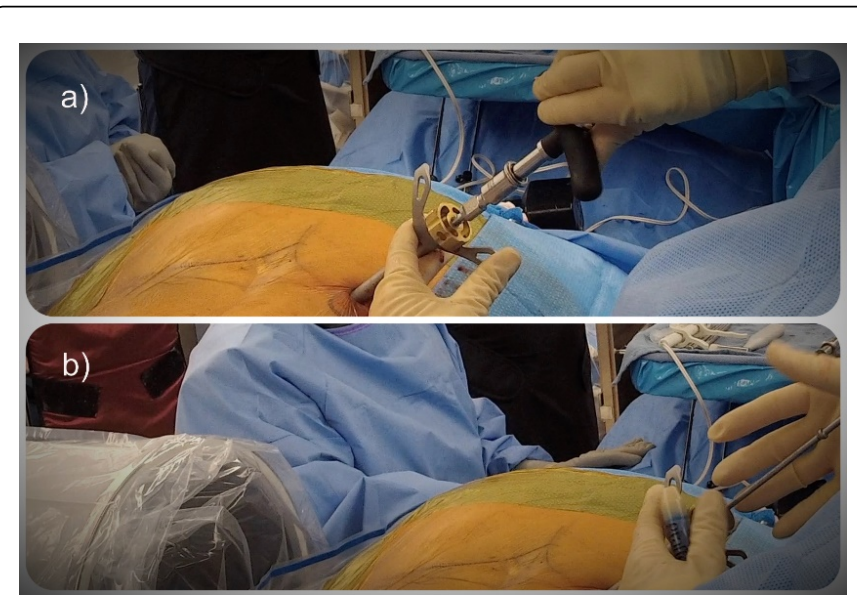

Figure 6: Intraoperative fluoroscopic view of final implant position in the anteroposterior (a), and lateral (b) plan. The lateral recess is decompressed. The probe can be passed around the L 5 pedicle (c). The view through the spinal endoscope above the expanded VariLift - $\mathrm{L}$ implant is filled with bone graft. The recessed implant and its relationship to the traversing and exiting nerve root is directly visualized with the spinal endoscope, d) endoscopic visualization of the placed VariLift" $\mathrm{L}$ implant including the endcap which is designed to contain the bone graft.

\section{Step 9: L5/S1 Fusion pearls}

Transforaminal access to the L5/S1 level for VariLift -L interbody fusion is typically no different in principle but can be more difficult if the access is obstructed either by a large sacral alar, or a large hypertrophic L5/S1 facet joint. Sacralization of the L5 vertebral body, or a high riding iliac crest, or the combination of the two factors may also dictate trajectories that are difficult to line up parallel to the $\mathrm{L} 5 / \mathrm{s} 1$ interspace. Typically, extensive bony resection of the obstructing portions of the sacral alar, and/or the L5/S1 facet joint complex are required. Care must be taken to stay above the intertransverse membrane to minimize bleeding that can otherwise be difficult to control. A high riding ilium can typically be dealt with by placing the skin incision higher and more posterolaterally. This author does not advocate for transiliac approach to the L5/S1 motion segment. Preoperative planning should focus on measuring the transforaminal approach trajectories to the L5/S1 neuroforamen both in the axial and coronal plane. Alternate approaches for L5/S1 interbody fusion should 
be chosen if the attack angles are greater than 60 degrees in any of the two planes.

These procedural steps typically allow dissection and decompression of the traversing and exiting nerve roots without overt retraction of the dorsal root ganglion, thereby increasing the volumetric size and reestablishing the axilla and the triangular safe zone between both roots and the inferior pedicle prior to preparation of the interspace with the sizing taps and insertion of the implant itself. The procedural steps of the percutaneous transforaminal endoscopic decompression and fusion are summarized in video 1 .

\section{Case Examples}

Of the 24 consecutive patients that underwent the endoscopic VARLIF procedure, 5 illustrative cases are described in the following.

\section{Case I}

An 80-year-old female patient was treated for foraminal and lateral recess stenosis at L4/L5 after having failed supportive non-operative, and interventional care with physical therapy, NSAIDs and epidural steroid injections (ESI) with --transforaminal endoscopic decompression procedures involving a foraminoplasty and microdiscectomy. During the decompression procedure the intervertebral disc was found to be void of any tissue and thus hollow consistent with preoperative MRI Pfirrmann Grade VI staging. Patient did well for about six months postoperatively but then developed recurrent radicular and mechanical low back pain symptoms insidiously. Ultimately, her walking endurance was reduced to similar distance as to prior the transforaminal decompression procedure, which prompted reimaging with MRI and plain film studies as well as supportive and interventional care measures. At this point, radiographic imaging studies showed Grade I spondylolisthesis and progressive vertical disc collapse and recurrent foraminal and lateral recess stenosis without disc extrusion and sequestration. The patient ultimately opted for surgical treatment. Patient underwent a vertical height restoration procedure with transforaminal oblique implantation of a single threaded $12 \mathrm{~mm} \varnothing$ VariLift'-L interbody fusion device placed in the center of the disc space. Local bone graft obtained during the foraminoplasty was mixed with demineralized allograft bone matrix. The bone graft was placed into the intervertebral disc space prior to cage insertion. Additional bone graft was placed through the center chamber of the implant and then extruded into the interspace via its lateral fenestrations. Intraoperative expansion of the device resulted in an overall foraminal height increase from $9 \mathrm{~mm}$ preoperatively to $23 \mathrm{~mm}$ postoperatively. The patient's visual analog scale (VAS) was reduced from 7.8 preoperatively to 2.6 postoperatively and walking endurance quadrupled. Patient reached opioid independence within two weeks after surgery. Imaging available up to six months postoperatively showed no signs of implant migration, subsidence, or expulsion into the neuroforamen. The intervertebral height remained unchanged.

\section{Case II}

A 62-year-old female was treated for symptomatic adjacent segment disease at L4/L5 following an L5/S1 TLIF some 10 years prior. The patient developed spondylolisthesis with associated symptomatic neurogenic claudication with radiculopathy, and decreased walking endurance. Patient opted for the VariLift ${ }^{\circ}-\mathrm{L}$ interbody fusion procedure after failed non-operative care to avoid lengthy and painful postoperative recovery and hospitalization associated with another TLIF operation involving supplemental posterior pedicle screw instrumentation at the adjacent level. The latter could be avoided as the surgery was done in an outpatient surgery setting. Using similar surgical implantation and grafting techniques as in case I the patient received a single threaded $12 \mathrm{~mm} \varnothing$ VariLift -L interbody fusion device. The overall foraminal height increase was $11 \mathrm{~mm}$ from $9 \mathrm{~mm}$ preoperatively to $20 \mathrm{~mm}$ postoperatively. The patient's VAS score was reduced from 6.9 preoperatively to 2.1 postoperatively. Patient required postoperative narcotic pain medication for five days. Postoperative imaging studies available up to four months postoperatively showed no signs of implant position with maintained neuroforaminal height.

\section{Case III}

A 39-year-old female was treated for L4/L5 isthmic spondylolisthesis that became symptomatic after TLIF at the L3/L4 level done for Grade II spondylolisthesis some two years prior. She had short-term relief of mechanical back and L4 radicular pain and soon thereafter presented with new-onset of L5 radicular pain that was first treated with non-operative and interventional care measures. The patient also received narcotic pain medication from a local pain management clinic. Dissatisfied with the side effects of opioids and her inability to return to work she opted for reconstructive fusion surgery at the L4/L5 level with the VariLift - L implant. Following implantation of a $12 \mathrm{~mm} \varnothing$ VariLift - L, the neuroforaminal height improved from $14 \mathrm{~mm}$ preoperatively to $24 \mathrm{~mm}$ postoperatively. The patient's VAS score was reduced from 6.9 pre-operatively to 2.1 postoperatively. Postoperative imaging studies available up to four months postoperatively showed no signs of implant position with maintained neuroforaminal height.

\section{Case IV}

A 69-year-old male patient developed recurrent claudication symptoms within a year after previous L4-S1 decompression and interventional neurectomy procedures. Patient was treated with a L4/L5 $11 \mathrm{~mm} \varnothing$ VariLift -L vertical height restoration procedure with local bone and demineralized allograft after having failed supportive non-operative, and interventional care with physical therapy, NSAIDs, and epidural steroid injections (ESI) resulting in overall foraminal height increase from $11 \mathrm{~mm}$ preoperatively to $19 \mathrm{~mm}$ postoperatively as measured on postoperative lateral lumbar radiographs. The patient's sequential preoperative plain film imaging studies showed progressive loss of intervertebral height and spondylolisthesis. Another lumbar MRI scan leading up to the VariLift -L surgery showed Pfirrmann stage $\mathrm{V}$ changes suggestive of advanced degenerative changes of the intervertebral disc material. During the decompression and intervertebral disc preparation portion of the procedure the intervertebral space was found to be void of any tissue and completely hollow from the annular entry point across the vertebral footprint to the opposite annulus. The patient's VAS score was reduced from 7.1 preoperatively to 3.6 postoperatively and walking endurance tripled. $\mathrm{He}$ reached opioid independence within five days after surgery. Postoperative radiographic surveillance follow-up studies available up to four months postoperatively showed no signs of implant migration, -subsidence, or expulsion into the neuroforamen. The intervertebral height remained unchanged. 


\section{Case V}

A 71-year-old female patient with postlaminectomy syndrome was treated with VariLift -L surgery for foraminal and lateral recess stenosis associated with facet arthropathy and spondylolisthesis at L4/L5 after having failed supportive non-operative and interventional care with physical therapy, NSAIDs, and epidural steroid injections (ESI). Preoperative MRI imaging showed Pfirrmann Grade VI at the L4/L5 level. Her walking endurance was reduced due to neurogenic claudication. The vertical height restoration accomplished with transforaminal oblique implantation of a single threaded $12 \mathrm{~mm} \varnothing$ VariLift - $L$ interbody fusion device produced an overall foraminal height increase from $10 \mathrm{~mm}$ preoperatively to $19 \mathrm{~mm}$ postoperatively. The patient's VAS score was reduced from 6.7 preoperatively to 3.1 postoperatively and walking endurance tripled. She reached opioid independence within four days from surgery. Imaging available up to five months postoperatively showed no implant- related problems. The intervertebral height remained unchanged.

\section{Tips, Pearls, Learning Curve, and Complications}

There were no immediate postoperative complications, superficial wound or deep infections, or readmissions to a hospital following the endoscopically assisted transforaminal VariLift ${ }^{\circ}$-L surgery. There were no intraoperative problems that prompted conversion to open surgery or abortion of the VariLift-L surgery. All patients were sent home from the recovery room. No patient Postoperative pain control was achieved with oral analgesics. Incisional pain was minimal in all patients and no patient required readmission to a hospital for postoperative pain control. However, dysethetic leg pain due to postoperative dorsal root ganglion irritation of the exiting nerve root during implantation was common and occurred in 14 of the 24 patients. They were treated with supportive care measures including tranforaminal epidural steroid injections which let to abatement of symptoms within less than 4 weeks. Implantation migration did not occur in any of the 24 patients during the available follow up of up to one year. However, minimal cage subsidence (less than $3 \mathrm{~mm}$ loss of height) occurred 7 of the 24 patients. These patients did not require any additional treatments. Complete L4/L5 cage subsidence occurred in one patient with osteoporosis. This patient was revised 3 months postop with an open decompression instrumented fusion surgery, which let to resolution of her recurrent symptoms. Another patient was treated with a repeat transforaminal decompression procedure 6 months postop from the VariLift ${ }^{\oplus}$ - surgery to deal with subsidence induced recurrent foraminal stenosis. This patient also did well with the follow up surgery.

As a result of a learning curve of a case series of 24 patients, the following tips and pearls appear important to note. First, a generous foraminoplasty with complete resection of the superior articular process should be performed to mobilize the spinal motion segment and facilitate the introduction of the implant. A partial pediculectomy and resection of obstructing osteophytes of the ring appophysis may aid in that. Second, endplate sparing decortication in preparation of the interbody fusion is critical to avoid excessive subsidence of the implant. Osteoporosis may exacerbate this problem. Paddle shavers used during the decortication maneuvers should rest on the opposite ring appophysis when rotated to avoid breaching the subchondral bone of the endplate. The inferior endplate is more susceptible to injury than the superior endplate during these maneuvers. Third, maximizing intervertebral height is critical to aid in indirect decompression of the foramen and lateral recess opposite from the access side. Under-sizing the implant may contribute to recurrent symptoms and should be avoided.

\section{Discussion and Conclusion}

This surgical technique guide shows that it is feasible to perform a percutaneous endoscopically assisted transforaminal decompression and fusion surgery with the expandable standalone VariLift - $\mathrm{L}$ interbody fusion system. This surgery is made possible by recent advances in videoendoscopic instrumentation allowing for aggressive bony decompression and resection maneuvers. Steps required to prepare the VariLift -L reconstruction of a diseased motion segment include foraminoplasty with resection of lateral overhang of a hypertrophic facet joint, osteotomy of the rostrally migrated SAP compressing the exiting nerve root in its axilla, partial pediculectomy, and resection of osteophytes of the ring apophysis to ease the entry of the conical tip of the cylindrical threaded cage.

The paddle shavers allow for easy distraction, mobilization, and removal of disc tissue during the discectomy, and endplate preparation during the outpatient transforaminal lumbar decompression surgery. This is particularly true in patients who have advanced degenerative disc disease with hollow intervertebral disc space. This distraction maneuver facilitates the indirect decompression by increasing neuroforaminal height and by minimizing the necessary retraction of the traversing and exiting nerve roots required to insert the cylindrical cage into the interspace. Preparation of the cage insertion site is facilitated by the extensile transforaminal bony decompression of the foraminal and lateral recess stenosis, which has been made feasible by the advanced endoscopes and decompression tools developed within the last five years such as reamers, trephines, motorized shavers, osteotomes, and rongeurs. These instruments afford better videoendoscopic visualization, tissue dissection, as well as more effective decompression maneuvers that were hitherto not possible. Surgical indications with spinal endoscopy, therefore, have broadened and are no longer limited to herniated disc. One example of this trend is the development of more specialized longer or shorter spinal endoscopes with larger central working channels in various oval or round configurations ranging from 4.1 to $6.9 \mathrm{~mm}$ (or larger) inner working channel diameter specifically designed for the transforaminal or direct posterior interlaminar approach, along with more robust optical, irrigation-, and suction systems that can tolerate the abuse caused during debridement by hammering, vibration, and vigorous repetitive high turnover cleaning and sterilization cycles. In addition, the maneuvering of the spinal endoscope in the epidural space (outside-in technique) [31] rather than inside the intervertebral disc (inside-out technique) [23-26] during the bony decompression procedure can cause epidural bleeding. The latter is better handled by modern and contemporary endoscopes that are more user friendly and can be tailored to individual surgeon requirements with various optical viewing angles. Expanded surgical indications have been demonstrated in previous literature for patients with advanced degenerative disc disease and associated lateral recess and foraminal stenosis due to advanced instability-induced degenerative changes of intervertebral disc and of the facet joint complex. However, no current literature was found demonstrating the implantation of an expandable cylindrical threaded interbody fusion cage during endoscopic transforaminal decompression procedure.

The importance of preoperative planning of transforaminal endoscopically assisted interbody fusion is equally important for removal of herniated discs. This has been stressed by Lee et al. who 
suggested a classification based on the location of a migrated disc fragment [32]. Other radiographic classification systems aiding in the preoperative decision making found to correlate with clinical outcomes have been published [32-35]. With respect to the expandable standalone VariLift $-\mathrm{L}$ interbody fusion system understanding of the spinal anatomy obstructing access to the intervertebral disc space it is critical to select suitable patients. A pre-operative CT scan as well as a bone density scan may aid in better patient selection and preoperative planning. In comparison to other lateral XLIF procedures, all levels of the lumbar spine can theoretically be treated surgically with the VariLift - L. Obviously, there is a learning curve to this procedure, and the novice surgeon should consider alternative interbody fusion procedures if the patient's reconstructive problem is outside hers or his comfort zone. Although the VariLift -L surgery could be completed in all of the 24 patients and none had to be converted to an open surgery, it is conceivable that the VariLift - L surgery cannot be completed from the transforaminal approach. In those cases, the same implant could theoretically still be used in a posterior lumbar interbody fusion surgery without pedicle screws. However, the advantage of the transforaminal approach would be lost.

\section{References}

1. Xie L, Wu WJ, Liang Y (2016) Comparison between minimally invasive transforaminal lumbar interbody fusion and conventional open transforaminal lumbar interbody fusion: An updated meta-analysis. Chin Med J 129: 1969-1986

2. Goldstein CL, Macwan K, Sundararajan K, Rampersaud YR (2016) Perioperative outcomes and adverse events of minimally invasive versus open posterior lumbar fusion: meta-analysis and systematic review. J Neurosurg Spine 24: 416-427.

3. Virdee JS, Nadig A, Anagnostopoulos G, George KJ (2017) Comparison of peri-operative and 12-month lifestyle outcomes in minimally invasive transforaminal lumbar interbody fusion versus conventional lumbar fusion. Br J Neurosurg 31: 167-171.

4. Phan K, Rao PJ, Kam AC, Mobbs RJ (2015) Minimally invasive versus open transforaminal lumbar interbody fusion for treatment of degenerative lumbar disease: A systematic review and meta-analysis. Eur Spine J 24: 1017-1030

5. Khan NR, Clark AJ, Lee SL, Venable GT, Rossi NB, et al. (2015) Surgical outcomes for minimally invasive vs open transforaminal lumbar interbody fusion: An updated systematic review and meta-analysis. Neurosurgery 77: 847-874.

6. Blumenthal SL, Ohnmeiss DD, NASS (2003) Intervertebral cages for degenerative spinal diseases. Spine J 3: 301-309.

7. Chen L, Yang H, Tang T (2005) Cage migration in spondylolisthesis treated with posterior lumbar interbody fusion using BAK cages. Spine 30: 2171-2175.

8. Choi JY, Sung KH (2006) Subsidence after anterior lumbar interbody fusion using paired stand-alone rectangular cages. Eur Spine J 15: 16-22.

9. Zdeblick TA, Phillips FM (2003) Interbody cage devices. Spine 28: 2-7.

10. Babu R, Park JG, Mehta AI, Shan T, Grossi PM, et al. (2012) Comparison of superior-level facet joint violations during open and percutaneous pedicle screw placement. Neurosurgery 71: 962-970.

11. Patel RD, Graziano GP, Vanderhave KL, Patel AA, Gerling MC (2011) Facet violation with the placement of percutaneous pedicle screws. Spine 36: 1749-1752.

12. Lee CS, Hwang CJ, Lee SW, Ahn YJ, Kim YT, et al. (2009) Risk factors for adjacent segment disease after lumbar fusion. Eur Spine J 18: 1637-1643.

13. Neely WF, Fichtel F, Del Monaco DC, Block JE (2016) Treatment of symptomatic lumbar disc degeneration with the VariLift-L interbody fusion system: Retrospective review of 470 cases. Int J Spine Surg 10: 15.

14. Barrett-Tuck R, Del Monaco D, Block JE (2017) One and two level posterior lumbar interbody fusion (PLIF) using an expandable, stand- alone, interbody fusion device: A VariLift ${ }^{t}$ case series. J Spine Surg 3: 9-15.

15. Emstad E, Del Monaco DC, Fielding LC, Block JE (2015) The VariLift $\left(^{\circ}\right)$ interbody fusion system: expandable, standalone interbody fusion. Med Devices 8: 219-230.

16. Ruetten S (2011) Full-endoscopic operations of the spine in disk herniations and spinal stenosis. Surg Technol Int 21: 284-298.

17. Komp M, Hahn P, Oezdemir S, Giannakopoulos A, Heikenfeld R, et al. (2015) Bilateral spinal decompression of lumbar central stenosis with the full-endoscopic interlaminar versus microsurgical laminotomy technique: A prospective, randomized, controlled study. Pain Physician 18: 61-70.

18. Komp M, Hahn P, Ozdemir S, Merk H, Kasch R, et al. (2014) Operation of lumbar zygoapophyseal joint cysts using a full-endoscopic interlaminar and transforaminal approach: prospective 2-year results of 74 patients. Surg Innov 21: 605-614.

19. Birkenmaier C, Komp M, Leu HF, Wegener B, Ruetten S (2013) The current state of endoscopic disc surgery: review of controlled studies comparing full-endoscopic procedures for disc herniations to standard procedures. Pain Physician 16: 335-344.

20. Sengupta DK, Herkowitz HN (2003) Lumbar spinal stenosis. Treatment strategies and indications for surgery. Orthop Clin North Am 34: 281-295.

21. Hoogland T, Schubert M, Miklitz B, Ramirez A (2006) Transforaminal posterolateral endoscopic discectomy with or without the combination of a low-dose chymopapain: a prospective randomized study in 280 consecutive cases. Spine 31: 890-897.

22. Schubert M, Hoogland T (2005) Endoscopic transforaminal nucleotomy with foraminoplasty for lumbar disk herniation. Oper Orthop Traumatol 17: 641-661.

23. Yeung AT, Yeung CA (2007) Minimally invasive techniques for the management of lumbar disc herniation. Orthop Clin North Am 38: 363-372.

24. Tsou PM, Alan Yeung C, Yeung AT (2004) Posterolateral transforaminal selective endoscopic discectomy and thermal annuloplasty for chronic lumbar discogenic pain: A minimal access visualized intradiscal surgical procedure. Spine J 4: 564-573.

25. Tsou PM, Yeung AT (2002) Transforaminal endoscopic decompression for radiculopathy secondary to intracanal noncontained lumbar disc herniations: outcome and technique. Spine J 2: 41-48.

26. Yeung AT, Yeung CA (2003) Advances in endoscopic disc and spine surgery: foraminal approach. Surg Technol Int 11: 255-263.

27. Kambin P, Casey K, O’Brien E, Zhou L (1996) Transforaminal arthroscopic decompression of lateral recess stenosis. J Neurosurg 84: $462-467$.

28. Kambin P, O'Brien E, Zhou L, Shaffer JL (1998) Arthroscopic microdiscectomy and selective fragmentectomy. Clin Orthop Relat Res 347: 150-167.

29. Kim MJ, Lee SH, Jung ES, Son BG, Choi ES, et al. (2007) Targeted percutaneous transforaminal endoscopic diskectomy in 295 patients: comparison with results of microscopic diskectomy. Surg Neurol 68: 623-631.

30. Ahn Y, Lee SH, Park WM, Lee HY, Shin SW, et al. (2014) Percutaneous endoscopic lumbar discectomy for recurrent disc herniation: surgical technique, outcome, and prognostic factors of 43 consecutive cases. Spine 29: 326-332.

31. Lewandrowski KU (2014) "Outside-in" technique, clinical results, and indications with transforaminal lumbar endoscopic surgery: a retrospective study on 220 patients on applied radiographic classification of foraminal spinal stenosis. Int J Spine Surg 8.

32. Lee S, Kim SK, Lee SH, Kim WJ, Choi WC, et al. (2007) Percutaneous endoscopic lumbar discectomy for migrated disc herniation: classification of disc migration and surgical approaches. Eur Spine J 16: 431-437.

33. Lee CK, Rauschning W, Glenn W (1998) Lateral lumbar spinal canal stenosis: classification, pathologic anatomy and surgical decompression. Spine 13: 313-320. 
Citation: Lewandrowski KU (2018) Surgical Technique of Endoscopic Transforaminal Decompression and Fusion with a Threaded Expandable Interbody Fusion Cage and A Report of 24 Cases. J Spine 7: 409. doi:10.4172/2165-7939.1000409

Page 8 of 8

34. Hasegawa T, An HS, Haughton VM, Nowicki BH (1995) Lumbar foraminal stenosis: Critical heights of the intervertebral discs and foramina. A cryomicrotome study in cadavera. J Bone Joint Surg Am 77: $32-38$.

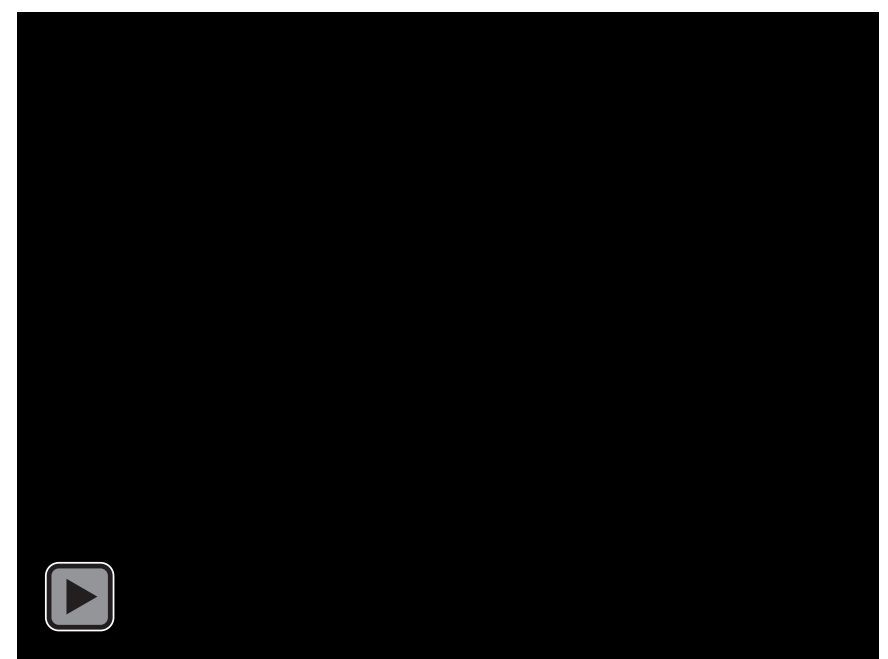

35. Macnab I (1971) Negative disc exploration: An analysis of the causes of nerve-root involvement in sixty-eight patients. J Bone Joint Surg Am 53: 891-903. 